\title{
Modelling Infectiology and Optimal Control of Dengue Epidemic
}

\author{
Laurencia Ndelamo Massawe ${ }^{1, ~}$, Estomih S. Massawe ${ }^{2}$, Oluwole Daniel Makinde ${ }^{3}$ \\ ${ }^{1}$ Faculty of Science, Technology and Environmental Studies, The Open University of Tanzania, Dar es Salaam, Tanzania \\ ${ }^{2}$ Mathematics Department, University of Dar es salaam, Dar es Salaam, Tanzania \\ ${ }^{3}$ Faculty of Military Science, Stellenbosch University, Saldanha, South Africa \\ Email address: \\ lndelamo@yahoo.com (L. N. Massawe)
}

\section{To cite this article:}

Laurencia Ndelamo Massawe, Estomih S. Massawe, Oluwole Daniel Makinde. Modelling Infectiology and Optimal Control of Dengue Epidemic. Applied and Computational Mathematics. Vol. 4, No. 3, 2015, pp. 181-191. doi: 10.11648/j.acm.20150403.21

\begin{abstract}
A mathematical model is presented to examine the interaction between human and vector populations. The model consists of five control strategies i.e. campaign aimed in educating careless individuals as a mean of minimizing or eliminating mosquito-human contact, control effort aimed at reducing mosquito-human contact, the control effort for removing vector breeding places, insecticide application and the control effort aimed at reducing the maturation rate from larvae to adult in order to reduce the number of infected individual. Optimal Control (OC) approach is used in order to find the best strategy to fight the disease and minimize the cost.
\end{abstract}

Keywords: Control, Optimal Control, Dengue Fever, Implementation, Strategy

\section{Introduction}

Optimal Control theory is a powerful Mathematical tool used to make decisions on how to control epidemiologic diseases like dengue fever disease. Optimal Control theory is used to minimize the investments in disease's control, since the financial resources are always scarce. Quantitative methods are applied to the optimization of investments in the control of the epidemiologic disease, in order to obtain a maximum of benefits from a fixed amount of financial resources [1]. Optimal control theory helps to find the percentage of the individuals who should be treated as time evolves in a given epidemic model in order to minimize the spread of disease and the cost of implementing the treatment strategy [2]. In dynamical system, the optimal control problem for ordinary differential equations is described by the state equation: $g_{i}^{\prime}(t)=q\left(t, g_{i}, u(t)\right)$ where $u(t)$ is control and

$g_{i}$ is the state variables which depend on the control variables. The control enters the system of differential equations and adjusts the dynamics of the state system. The goal is to adjust the control in order to maximize (or minimize) a given objective function subject to some constraints [2].The aim of the control is to minimize the objective function i.e.

$$
J=\min _{u} \int_{t_{0}}^{t}\left(t, g_{i}, u(t)\right) d t
$$

subject to the differential equations and initial conditions. Such a minimizing control is called an optimal control problem [2].

The principle technique for such an optimal control problem is to solve a set of "necessary conditions" that an optimal control and corresponding state variables must satisfy. The necessary conditions is generated from the Hamiltonian $H$, which is defined as

$$
H\left(t, g_{i}, u, \lambda\right)=f\left(t, \mathrm{~g}_{i}, u\right)+\lambda q\left(t, g_{i}, u\right)
$$

subject to

$$
\frac{d g_{i}}{d t}=q\left(g_{i}(t), u(t), t\right)
$$

$$
\Rightarrow H=\text { integrand of (1) }+ \text { adjoint * Right hand side (RHS) of (2). }
$$


Then it is intended to minimize $H$ with respect to $u=u^{*}$ (optimal control) and the conditions is written in terms of the Hamiltonian:

$$
\begin{gathered}
\frac{\partial H}{\partial u}=0 \text { at } u^{*} \Rightarrow f_{u}+\lambda q_{u}=0 \text { (Optimality condition), } \\
\frac{d \lambda}{d t}=-\frac{\partial H}{\partial g_{i}} \Rightarrow \frac{d \lambda}{d t}=-\left(f_{g_{i}}+\lambda q_{g_{i}}\right) \text { (Adjoint equation), } \\
\lambda\left(t_{1}\right)=0 \text { (Transversality condition), }
\end{gathered}
$$

The dynamics of the state equation is given by

$$
g_{i}^{\prime}=q\left(t, g_{i}, u\right)=\frac{\partial H}{\partial \lambda}, \mathrm{g}\left(t_{0}\right)=g_{0} \text { in [2] }
$$

Dengue fever (DF) is a vector-borne disease transmitted by female Aedes aegypti and Aedes albopictus mosquitoes. Dengue fever (DF) is still endemic in many countries. Dengue fever is one of the diseases that have claimed and continue to claim the lives of millions of people worldwide. Currently 2.5 billion people living in areas at risk of DF transmission, each year, an estimated 100 million cases of dengue fever occur worldwide[3,4]. The disease create many burdens on families as some bread winners and also the governments which have to spent millions of money in diagnosing the disease, purchasing of pesticides to kill the mosquitoes and purchasing drugs to treat the patients and the other intervention schemes. It is these effects of the disease that call for continuous research into the prevention and control of the disease.

Mathematical models have played a major role in increasing our understanding of the dynamics of infectious diseases. Several models have been proposed to study the effects of some factors on the transmission dynamics of these infectious diseases including Dengue fever and to provide guidelines as to how the spread can be controlled [5].

Mathematical modelling also became considerable important tool in the study of epidemiology because it helps us to understand the observed epidemiological patterns, disease control and provide understanding of the underlying mechanisms which influence the spread of disease and may suggest control strategies [6], The epidemiological data and the economic cost of infectious diseases are effective elements in evaluating the relevance of intervention programmes. In economic situation, any intervention, like treatment, that has been found to be cost effective would be fully funded without delay. Sometimes, funding and access to treatment may be difficult as always faced with a number of constraints. Optimal control theory to determine the optimal resource allocation as an epidemic progress has been used. Optimal control theory is a powerful mathematical tool to make decision involving complex dynamical systems [2]. For example, what percentage of the population should be treated as time evolves in a given epidemic model to minimize both the number of infected people and the cost of implementing the treatment strategies. The desired outcome depends on the particular situation. New drug treatments and combinations of drugs are under constant development.

Optimal control problems have generated a lot of interest from researchers all over the world, for instance in [7], the authors presented a mathematical model of optimal control by considering the cost of insecticide application, the cost of the production of irradiated mosquitoes and their delivery as well as the social cost. In [8], the authors used three vector control tools: larvicide, adulticide and mechanical control, where the problem is studied using an Optimal Control (OC) approach. In [9], the authors presented an application of optimal control theory to Dengue epidemics. The dynamic model is described by a set of nonlinear ordinary differential equations that depend on the dynamics of the Dengue mosquito, the number of infected individuals, and the people's motivation to combat the mosquito. The cost functional depends not only on the costs of medical treatment of the infected people but also on the costs related to educational and sanitary campaigns. They used two approaches to solve the problem: one using optimal control theory, another one by discretizing first the problem and then solving it with nonlinear programming, leading to a decrease of infected mosquitoes and individuals in less time and with lower costs. In [10], the authors used optimal control theory for control of the vector i.e. mosquito. Their model consists of eight mutually exclusive compartments representing the human and vector dynamics. It also includes a control parameter (insecticide) in order to fight the mosquito. In [6], the authors used the optimal control theory in which their model consists of three control measures; the preventive control to minimize vector human contacts, the treatment control to the infected human, and the insecticide control to the vector. In [11], the authors used the optimal vaccination strategies to minimize the susceptible and infected individuals and to maximize the number of recovered individuals. In [12], the authors used two controls representing the effort that reduces the contact between the infectious and susceptible individuals, and a therapeutic treatment. The objective function was based on a combination of minimizing the number of latent and infected individuals and the cost. The optimal controls were obtained by solving the optimality system. In [13], the authors investigated the effectiveness and optimal control strategies of indoor residual spraying (IRS), insecticide treated nets (ITNs) and treatment on the transmission dynamics of malaria in Karonga District, Malawi.

Not much research has been done in the study of epidemic models that consider the optimal control to reduce the spread of the dengue fever disease through the campaign to educate the careless human susceptible, control mosquitoes, removing vector breeding places or the use of gene modifying mosquito where the offspring die before being matured. Moreover in [14], the authors presented a dynamical model that studied the temporal model for dengue disease with treatment. In this work, the model by [14] will be extended, to include temporary immunity, optimal control analysis and Susceptibles with different behaviour i.e. the dynamical system that incorporates the effects of Careful and Careless 
human susceptible on the transmission of Dengue fever in the society.

The optimal levels of various intervention strategies needed to optimally reduce the spread of the disease through the campaign to educate the careless human susceptible, control mosquitoes bite, by using mosquito nets or mosquito repellent, removing vector breeding places and the social cost (Social cost means all the expenses related to the disease like infectives treatment, hospital care and death) will be studied.

The basic model to include various intervention strategies to obtain an optimal control problem will be analysed qualitatively using the Pontryagin's Maximum principle. The resulting optimal control problem is also solved numerically to gain more insights into the implications of the interventions.

\section{Model Formulation}

In this paper hosts and vectors are considered. The hosts (humans) are divided into five mutually-exclusive compartments, namely, $S_{h_{1}}$-Careful human Susceptibles, $S_{h_{2}}$ -Careless human Susceptibles, $I_{h}$-infected human, $T_{h}$ treated infected human , $R_{h}$ - recovery infected human, Similarly, the model has three compartments for the vectors (mosquitoes) indexed by $m$ given by: $A_{m}$-Aquatic phase (that includes the egg, larva and pupa stages), $S_{m}$ - Susceptibles (mosquitoes that are able to contract the disease), $I_{m}$ -Infectives (mosquitoes capable of transmitting the disease to human) [15].Then we consider five controls:

$u_{1}$ is the control effort aimed at changing behaviour of careless human susceptible i.e. $u_{1}$ is the campaign aimed in educating careless individual $\left(u_{1}\right)$ as a means of minimizing or eliminating mosquito-human contact, $u_{2}$ is the control effort aimed at reducing mosquito-human contact, $u_{3}$ represent the control measure for removing vector breeding places , $u_{4}$ represent insecticide application and $u_{5}$ is the control effort aimed at reducing the maturation rate from larvae to adult. These control functions $u_{1}, u_{2}, u_{3}, u_{4}$ and $u_{5}$ are bounded and Lebesgue integrable.

In formulating the model, the following assumptions are considered [15]:

i. Total human population $\left(N_{h}\right)$ is constant,

ii. The population is homogeneous, which means that every individual of a compartment is homogeneously mixed with the other individuals, immigration and emigration are not considered,

iii. Each vector has an equal probability to bite any host,

iv. Humans and mosquitoes are assumed to be born susceptible i.e. there is no natural protection,

v. The coefficient of transmission of the disease is fixed and does not vary seasonally,

vi. For the mosquito there is no resistant phase, due to its short lifetime,

vii. The biting rate of careless human susceptible is higher than that for careful human susceptible.

Considering the above considerations and assumptions, we then have the following schematic model flow diagram for dengue fever disease with control

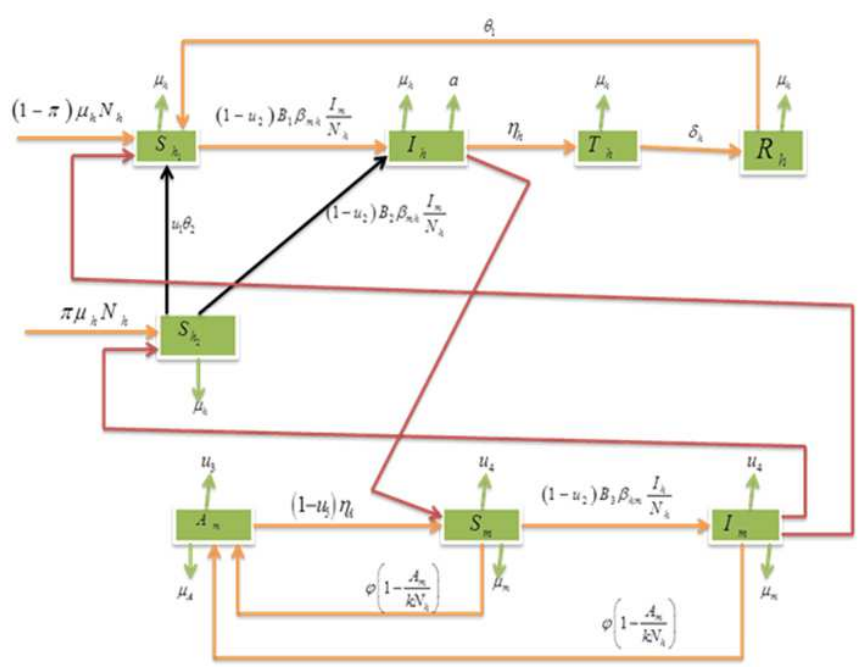

Figure 1. Model Flow diagram for dengue fever disease with control.

From the above flow diagram, the model will be governed by the following equations [15]:

$$
\begin{gathered}
\frac{d S_{h_{1}}}{d t}=(1-\pi) \mu_{h} N_{h}-\left(1-u_{2}\right) B_{1} \beta_{m h} \frac{I_{m}}{N_{h}} S_{h_{1}}-\mu_{h} S_{h_{1}}+\theta_{1} R_{h}+u_{1} \theta_{2} S_{h_{2}}, \\
\frac{d S_{h_{2}}}{d t}=\pi \mu_{h} N_{h}-\left(1-u_{2}\right) B_{2} \beta_{m h} \frac{I_{m}}{N_{h}} S_{h_{2}}-\mu_{h} S_{h_{2}}-u_{1} \theta_{2} S_{h_{2}}, \\
\frac{d I_{h}}{d t}=\left(\left(1-u_{2}\right) B_{1} S_{h_{1}}+\left(1-u_{2}\right) B_{2} S_{h_{2}}\right) \beta_{m h} \frac{I_{m}}{N_{h}}-\left(\mu_{h}+\eta_{h}+a\right) I_{h}, \\
\frac{d T_{h}}{d t}=\eta_{h} I_{h}-\left(\mu_{h}+\delta_{h}\right) T_{h}, \\
\frac{d R_{h}}{d t}=\delta_{h} T_{h}-\left(\mu_{h}+\theta_{1}\right) R_{h}, \\
\frac{d A_{m}}{d t}=\varphi\left(1-\frac{A_{m}}{k N_{h}}\right)\left(S_{m}+I_{m}\right)-\left(\mu_{A}+\left(1-u_{5}\right) \eta_{A}+u_{3}\right) A_{m}, \\
\frac{d S_{m}}{d t}=\left(1-u_{5}\right) \eta_{A} A_{m}-\left(\left(1-u_{2}\right) B_{3} \beta_{h m} \frac{I_{h}}{N_{h}}+\mu_{m}\right) S_{m}-u_{4} S_{m}
\end{gathered}
$$

and

$$
\frac{d I_{m}}{d t}=\left(1-u_{2}\right) B_{3} \beta_{h m} \frac{I_{h}}{N_{h}} S_{m}-\left(\mu_{m}+u_{4}\right) I_{m} .
$$

\section{Model Analysis}

In this section the approach similar to [5] is used. The aim is to seek optimal levels of the intervention strategies needed to minimize the number of infected human and the cost of 
implementing the control strategies. A functional $J$ given by

$$
\begin{aligned}
J= & \min _{u_{i}, i \in[1,5]} \int_{0}^{T}\left(A_{1} S_{h_{1}}(t)+A_{2} S_{h_{2}}(t)+A_{3} I_{h}(t)+A_{4} I_{m}(t) d t\right. \\
& \left.+\frac{1}{2} D_{3} u_{3}^{2}+\frac{1}{2} D_{4} u_{4}^{2}+\frac{1}{2} D_{5} u_{5}^{2}\right) d t
\end{aligned}
$$

subject to the differential equations (3) and initial condition chosen as. $S_{h_{1}}(0) \geq 0, S_{h_{2}}(0) \geq 0, I_{h}(0) \geq 0, T_{h}(0) \geq 0$, $R_{h}(0) \geq 0, A_{m}(0) \geq 0, S_{m}(0) \geq 0$ and $I_{m}(0) \geq 0$ where the $D_{i} s$ are positive weights which measure relative costs of implementing the respective intervention strategies over the period $[0, T]$, whilst the terms $\frac{D_{i} u_{i}{ }^{2}}{2}$ measure the cost of the intervention strategies, $A_{i}$ is the positive weights, $S_{h_{1}}(t)$ is careful human Susceptibles, $S_{h_{2}}(t)$ is careless human Susceptibles, $I_{h}$ is the infected human, $I_{m}$ is the infected

$$
\begin{gathered}
H=A_{1} S_{h_{1}}+A_{2} S_{h_{2}}+A_{3} I_{h}+A_{4} I_{m}+\frac{1}{2} D_{1} u_{1}^{2}+\frac{1}{2} D_{2} u_{2}^{2}+\frac{1}{2} D_{3} u_{3}^{2}+\frac{1}{2} D_{4} u_{4}^{2}+\frac{1}{2} D_{5} u_{5}^{2}+ \\
+\lambda_{1}\left[(1-\pi) \mu_{h} N_{h}-\left(1-u_{2}\right) B_{1} \beta_{m h} \frac{I_{m}}{N_{h}} S_{h_{1}}-\mu_{h} S_{h_{1}}+\theta_{1} R_{h}+u_{1} \theta_{2} S_{h_{2}}\right]+\lambda_{2}\left[\pi \mu_{h} N_{h}-\left(1-u_{2}\right) B_{2} \beta_{m h} \frac{I_{m}}{N_{h}} S_{h_{2}}-\mu_{h} S_{h_{2}}-u_{1} \theta_{2} S_{h_{2}}\right] \\
+\lambda_{3}\left[\left(\left(1-u_{2}\right) B_{1} S_{h_{1}}+\left(1-u_{2}\right) B_{2} S_{h_{2}}\right) \beta_{m h} \frac{I_{m}}{N_{h}}-\left(\mu_{h}+\eta_{h}+a\right) I_{h}\right]+\lambda_{4}\left[\eta_{h} I_{h}-\left(\mu_{h}+\delta_{h}\right) T_{h}\right]+\lambda_{5}\left[\delta_{h} T_{h}-\left(\mu_{h}+\theta_{1}\right) R_{h}\right] \\
+\lambda_{6}\left[\varphi\left(1-\frac{A_{m}}{k N_{h}}\right)\left(S_{m}+I_{m}\right)-\mu_{A} A_{m}-u_{3} A_{m}-\left(1-u_{5}\right) \eta_{A} A_{m}\right]+\lambda_{h}\left[\left(1-u_{5}\right) \eta_{A} A_{m}-\left(\left(1-u_{2}\right) B_{3} \beta_{h m} \frac{I_{h}}{N_{h}}+\mu_{m}\right) S_{m}-u_{4} S_{m}\right] \\
+\lambda_{8}\left[\left(1-u_{2}\right) B_{3} \beta_{h m} \frac{I_{h}}{N_{h}} S_{m}-\left(\mu_{m}+u_{4}\right) I_{m}\right]
\end{gathered}
$$

where $\lambda_{i}(i=1,2, \ldots, 8)$ are the adjoint variables or co-state variables which determine the adjoint system, together with the state system (3) describes the optimality system. Pontryagin's Maximum principle [17] and the existence result for optimal control from [18] can be used to obtain the following proposition.

Proposition 1. The optimal control 5-tuple $\left(u_{1}, u_{2}, u_{3}, u_{4}, u_{5}\right)$ minimizes the functional $J$ if there exist mosquito and $i$ is the number of controls applied to the system, $T$ is the final time.

Quadratic cost on the controls is preferred and this is similar with what is in other literature on epidemic controls [16]. The goal is to minimize infection, while minimizing the cost of

Thus, we seek an optimal control $u^{*}$ such that

$$
J\left(u^{*}\right)=\min \{J(u) \mid u \in U\}
$$

where $U$ is the control set defined by $U=\left\{\left(u_{1}, \ldots \ldots \ldots, u_{5}\right)\right.$ such that $u_{i}$ are measurable with $0 \leq u_{i}(t) \leq 1 ; \forall t \quad \mathcal{E}$ $[0, T]\}$ is the set of admissible controls. The necessary conditions that an optimal must satisfy come from Pontryagin's Maximum Principle[17].This principle converts a dynamical system (differential equation of the $i^{\text {th }}$ state variable ) and (4) above into a problem of minimizing pointwise a Hamiltonian $H$, with respect to $u$ where;$$
\text { adjoint variables } \lambda_{i}, i=1,2, \ldots ., 8 \text { that satisfy the adjoint }
$$$$
\text { system given by }
$$$$
\frac{d \lambda_{1}}{d t}=-\frac{\partial H}{\partial S_{h_{1}}} .
$$

But from (6),

$$
\frac{\partial H}{\partial S_{h_{1}}}=A_{1}+\lambda_{1}\left[-\left(1-u_{2}\right) B_{1} \beta_{m h} \frac{I_{m}}{N_{h}}-\mu_{h}\right]+\lambda_{3}\left[\left(1-u_{2}\right) B_{1}\right] \beta_{m h} \frac{I_{m}}{N_{h}}
$$

Hence

$$
\frac{d \lambda_{1}}{d t}=-A_{1}+\lambda_{1}\left[\left(1-u_{2}\right) B_{1} \beta_{m h} \frac{I_{m}}{N_{h}}+\mu_{h}\right]-\lambda_{3}\left[\left(1-u_{2}\right) B_{1}\right] \beta_{m h} \frac{I_{m}}{N_{h}}
$$

Others will be obtained using the same method. Therefore 


$$
\begin{gathered}
\frac{d \lambda_{2}}{d t}=-A_{2}-\lambda_{1}\left[u_{1} \theta_{2}\right]+\lambda_{2}\left[\left(1-u_{2}\right) B_{2} \beta_{m h} \frac{I_{m}}{N_{h}}+\mu_{h}+u_{1} \theta_{2}\right]-\lambda_{3}\left[\left(1-u_{2}\right) B_{2} \beta_{m h} \frac{I_{m}}{N_{h}}\right] \\
\frac{d \lambda_{3}}{d t}=-A_{3}+\lambda_{3}\left[\mu_{h}+\eta_{h}+a\right]-\lambda_{4} \eta_{h}+\lambda_{7}\left[\left(1-u_{2}\right) B_{3} \beta_{h m} \frac{S_{m}}{N_{h}}\right]-\lambda_{8}\left[\left(1-u_{2}\right) B_{3} \beta_{h m} \frac{S_{m}}{N_{h}}\right], \\
\frac{d \lambda_{4}}{d t}=\lambda_{4}\left(\mu_{h}+\delta_{h}\right)-\lambda_{5} \delta_{h}, \frac{d \lambda_{5}}{d t}=-\lambda_{1} \theta_{1}+\lambda_{5}\left(\mu_{h}+\theta_{1}\right), \\
\frac{d \lambda_{6}}{d t}=\lambda_{6}\left[\frac{\varphi}{k N_{h}}\left(S_{m}+I_{m}\right)+\left(\mu_{A}+u_{3}+\left(1-u_{5}\right) \eta_{A}\right)\right]-\lambda_{7}\left(1-u_{5}\right) \eta_{A}, \\
\frac{d \lambda_{7}}{d t}=-\lambda_{6}\left[\varphi\left(1-\frac{A_{m}}{k N_{h}}\right)\right]+\lambda_{7}\left[\left(\left(1-u_{2}\right) B_{3} \beta_{h m} \frac{I_{h}}{N_{h}}+\mu_{m}\right)+u_{4}\right]-\lambda_{8}\left[\left(1-u_{2}\right) B_{3} \beta_{h m} \frac{I_{h}}{N_{h}}\right]
\end{gathered}
$$

and

$$
\begin{gathered}
\frac{d \lambda_{8}}{d t}=-A_{4}+\lambda_{1}\left[\left(1-u_{2}\right) B_{1} \beta_{m h} \frac{S_{h_{1}}}{N_{h}}\right]+\lambda_{2}\left[\left(1-u_{2}\right) B_{2} \beta_{m h} \frac{S_{h_{2}}}{N_{h}}\right] \\
-\lambda_{3}\left[\left(\left(1-u_{2}\right) B_{1} S_{h_{1}}+\left(1-u_{2}\right) B_{2} S_{h_{2}}\right) \frac{\beta_{m h}}{N_{h}}\right]-\lambda_{6}\left[\varphi\left(1-\frac{A_{m}}{k N_{h}}\right)\right]+\lambda_{8}\left(\mu_{m}+u_{4}\right) .
\end{gathered}
$$

To get the characterization of the optimal control we solve the Equations $\frac{\partial H}{\partial u_{i}}=0$ at $u_{i}=u_{i}^{*}$ where $i=1,2, \ldots n$ and $n$ is number of controls. The first control is obtained as

$$
\frac{\partial H}{\partial u_{1}}=D_{1} u_{1}+\lambda_{1}\left[\theta_{2} S_{h_{2}}\right]-\lambda_{2}\left[\theta_{2} S_{h_{2}}\right]
$$

from (6)

$$
\begin{aligned}
& \text { Then we set } \frac{\partial H}{\partial u_{1}}=0 \text { to get } \\
& \qquad D_{1} u_{1}+\lambda_{1}\left[\theta_{2} S_{h_{2}}\right]-\lambda_{2}\left[\theta_{2} S_{h_{2}}\right]=0
\end{aligned}
$$

$$
\begin{gathered}
u_{2}=\left\{\begin{array}{c}
\lambda_{3}\left[\left(B_{1} S_{h_{1}}+B_{2} S_{h_{2}}\right) \beta_{m h} \frac{I_{m}}{N_{h}}\right]+\lambda_{8}\left[B_{3} \beta_{h m} \frac{I_{h}}{N_{h}} S_{m}\right]- \\
\lambda_{1}\left[B_{1} \beta_{m h} \frac{I_{m}}{N_{h}} S_{h_{h}}\right]-\lambda_{2}\left[B_{2} \beta_{m h} \frac{I_{m}}{N_{h}} S_{h_{2}}\right]-\lambda_{1}\left[B_{3} \beta_{h m} \frac{I_{h}}{N_{h}} S_{m}\right]
\end{array}\right] / D_{2}, \\
u_{3}=\frac{\lambda_{6} A_{m}}{D_{3}}, u_{4}=\frac{\lambda_{7} S_{m}+\lambda_{8} I_{m}}{D_{4}}
\end{gathered}
$$

and

$$
u_{5}=\frac{\eta_{A} A_{m}\left(-\lambda_{6}+\lambda_{7}\right)}{D_{5}}
$$

or

$$
D_{1} u_{1}=-\theta_{2} S_{h_{2}} \lambda_{1}+\theta_{2} S_{h_{2}} \lambda_{2}
$$

Consequently $u_{1}=\frac{-\theta_{2} S_{h_{2}} \lambda_{1}+\theta_{2} S_{h_{2}} \lambda_{2}}{D_{1}}$

Other controls for $u_{2} \quad u_{3} u_{4}$ and $u_{5}$ are obtained similarly.

In order to satisfy the given bounds for the control functions, i.e. $0 \leq u(t) \leq 1$ and $t \in[0, T]$ the optimal control is restricted to $u_{i}^{*}=\min \left\{1, \max \left(0, u_{i}\right)\right\}$. Therefore

Thus

$$
u_{1}^{*}=\min \left\{1, \max \left(0, \frac{-\theta_{2} S_{h_{2}} \lambda_{1}+\theta_{2} S_{h_{2}} \lambda_{2}}{D_{1}}\right)\right\},
$$

$$
\left.u_{2}^{*}=\min \left\{1, \max \left(0,\left\{\begin{array}{l}
\lambda_{3}\left[\left(B_{1} S_{h_{1}}+B_{2} S_{h_{2}}\right) \beta_{m h} \frac{I_{m}}{N_{h}}\right]+\lambda_{8}\left[B_{3} \beta_{h m} \frac{I_{h}}{N_{h}} S_{m}\right]- \\
\lambda_{1}\left[B_{1} \beta_{m h} \frac{I_{m}}{N_{h}} S_{h_{1}}\right]-\lambda_{2}\left[B_{2} \beta_{m h} \frac{I_{m}}{N_{h}} S_{h_{2}}\right]-\lambda_{7}\left[B_{3} \beta_{h m} \frac{I_{h}}{N_{h}} S_{m}\right]
\end{array}\right\}\right) / D_{2}\right)\right\},
$$




$$
u_{3}^{*}=\min \left\{1, \max \left(0, \frac{\lambda_{6} A_{m}}{D_{3}}\right)\right\}, u_{4}^{*}=\min \left\{1, \max \left(0, \frac{\lambda_{7} S_{m}+\lambda_{8} I_{m}}{D_{4}}\right)\right\}
$$

and

$$
u_{5}^{*}=\min \left\{1, \max \left(0, \frac{\eta_{A} A_{m}\left(-\lambda_{6}+\lambda_{7}\right)}{D_{5}}\right)\right\}
$$

\section{Numerical Simulation}

In this section, we illustrate the analytical results of the study by carrying out numerical simulations of the model system (3) and study the effects of campaign to educate the careless human susceptible $\left(u_{1}\right)$, control vector-human contact $\left(u_{2}\right)$, removing vector breeding areas $\left(u_{3}\right)$, insecticides application $\left(u_{4}\right)$ and control maturation rate from larvae to adult $\left(u_{5}\right)$. We investigate and compare numerical results in the following strategies:

(i) when all controls are set to zero, (ii) When insecticides application $\left(u_{4}\right)$ is used to optimize the objective function $(J)$ while other controls are set to zero, (iii) When removing vector breeding areas $\left(u_{3}\right)$, insecticides application $\left(u_{4}\right)$ and control maturation rate from larvae to adult $\left(u_{5}\right)$ are used to optimize the objective function $(J)$ while other controls are set to zero, (iv) When campaign to educate the careless human susceptible $\left(u_{1}\right)$, removing vector breeding areas $\left(u_{3}\right)$, insecticides application $\left(u_{4}\right)$ and control maturation rate from larvae to adult $\left(u_{5}\right)$ are used to optimize the objective function $(J)$ while control vector-human contact $\left(u_{2}\right)$ is set to zero, (v) when control vector human contact $\left(u_{2}\right)$ and insecticides application $\left(u_{4}\right)$ are used to optimize the objective function $(J)$ while other controls are set to zero, (vi) When only control vector- human contact $\left(u_{2}\right)$ is used to optimize the objective function $(J)$ while other controls are set to zero, (vii)When campaign to educate the careless human susceptible $\left(u_{1}\right)$ and control vector-human contact $\left(u_{2}\right)$ are used to optimize the objective function $(J)$ while other controls are set to zero and (viii) When all controls are used to optimize the objective function $(J)$. Parameter values are obtained from the different literatures like (http://www.wavuti.com/2014/05 /wizara- ya -afya- kitengo -cha.html), [14], [15] and [19], other parameter values are estimated to vary within realistic means and given as shown below $\beta_{h m}=0.375, \beta_{m h}=0.45, \pi=0.96, \quad B_{1}=0.5$, $B_{2}=0.9, \quad B_{3}=0.7, \quad \mu_{m}=\frac{1}{11}, k=3, \quad \eta_{A}=0.35$, $\mu_{A}=0.25, \mu_{h}=\frac{1}{78 \times 365}, \eta_{h}=1 / 3, \varphi=5, \theta_{2}=0.6$, $a=0.001, \mathrm{~N}_{1}=20, \mathrm{~A}_{1}=10, \mathrm{~A}_{2}=15, \mathrm{~A}_{3}=20$, $\mathrm{A}_{4}=25, \mathrm{D}_{1}=50, \mathrm{D}_{2}=60, \mathrm{D}_{3}=80, \mathrm{D}_{4}=10$ and
$\mathrm{D}_{5}=15$ (7) Figures 2 (i)-(viii) show the effects of optimal control strategies on the spread of dengue fever disease in the population.

This shows effects of optimal control of the model system (3) using the parameter values in (7) for different strategies as shown below.

$$
\begin{aligned}
& \text { 1. } u_{1}=u_{2}=u_{3}=u_{4}=u_{5}=0, \\
& \text { 2. } u_{2} \neq 0, u_{1}=u_{3}=u_{4}=u_{5}=0, \\
& \text { 3. } u_{4} \neq 0, u_{1}=u_{2}=u_{3}=u_{5}=0, \\
& \text { 4. } u_{1} \neq 0, u_{2} \neq 0, u_{3}=u_{4}=u_{5}=0, \\
& \text { 5. } u_{1}=0, u_{2}=0, u_{3} \neq 0, u_{4} \neq 0, u_{5} \neq 0, \\
& \text { 6. } u_{1} \neq 0, u_{2}=0, u_{3} \neq 0, u_{4} \neq 0, u_{5} \neq 0, \\
& \text { 7. } u_{2} \neq 0, u_{4} \neq 0, u_{1}=u_{3}=u_{5}=0, \\
& \text { 8. } u_{1} \neq 0, u_{2} \neq 0, u_{3} \neq 0, u_{4} \neq 0, u_{5} \neq 0, \\
& \text { 9. } u_{1}=u_{2}=u_{3}=u_{4}=u_{5}=0 .
\end{aligned}
$$
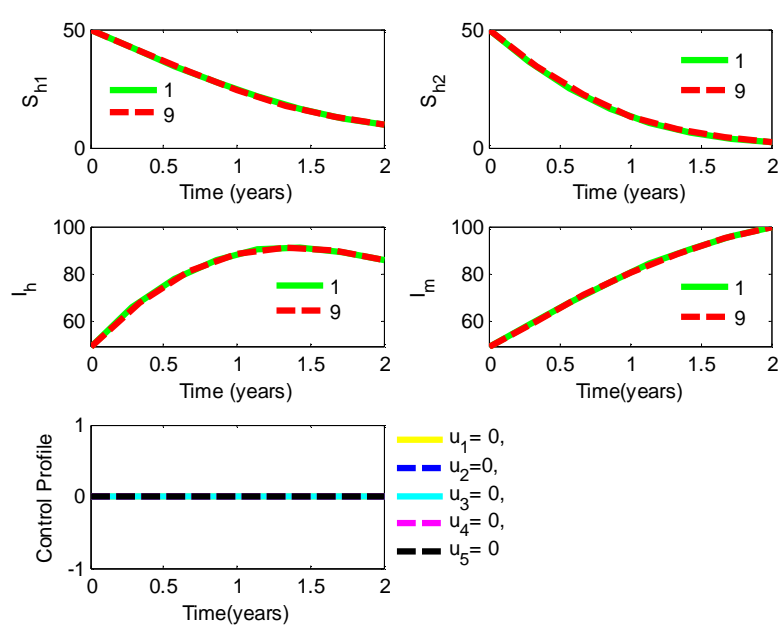

(i)
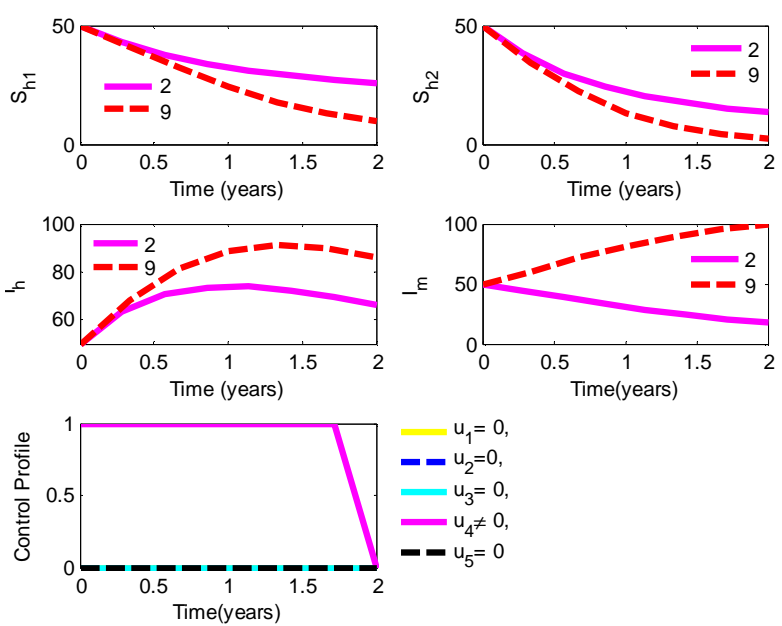

(ii) 

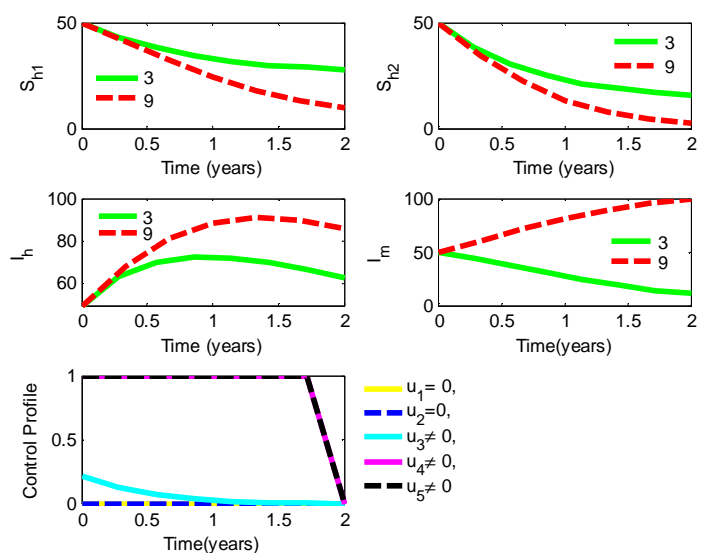

(iii)
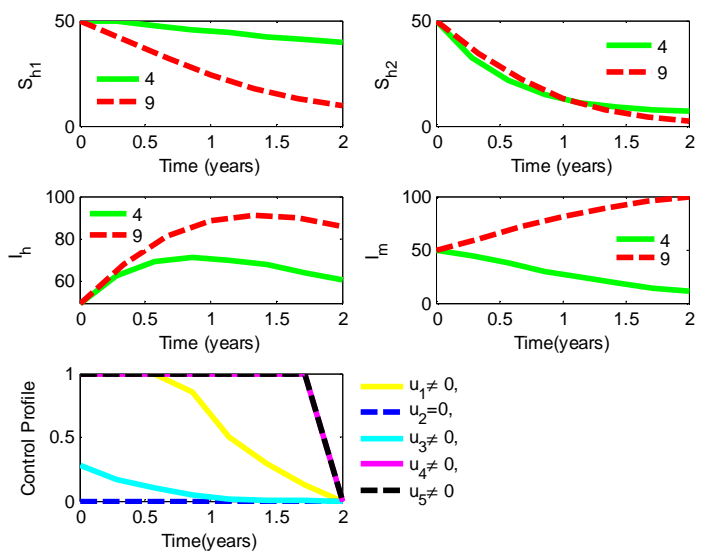

(iv)
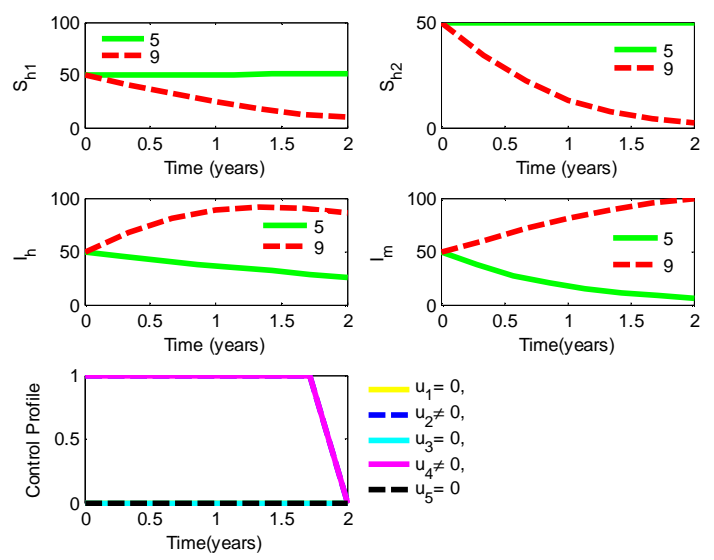

(v)

From figure 2(i) it is observed that when no control is applied careful and careless human susceptible population decrease and infected human and mosquito population increases. The control profile shows that all controls are in a lower bound.

From figure 2(ii)-(viii) it is observed that careful and careless human susceptible population increased while
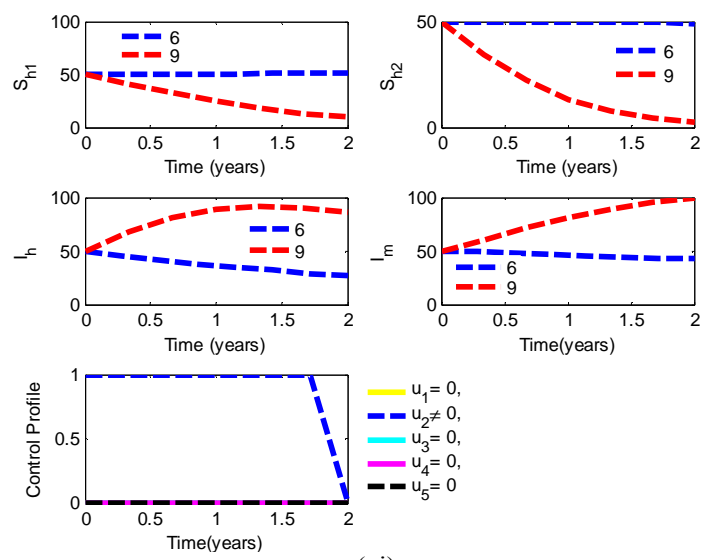

(vi)
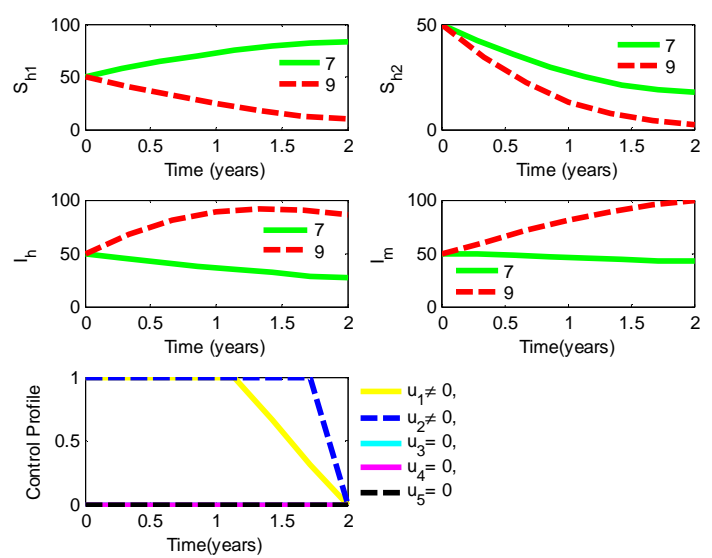

(vii)
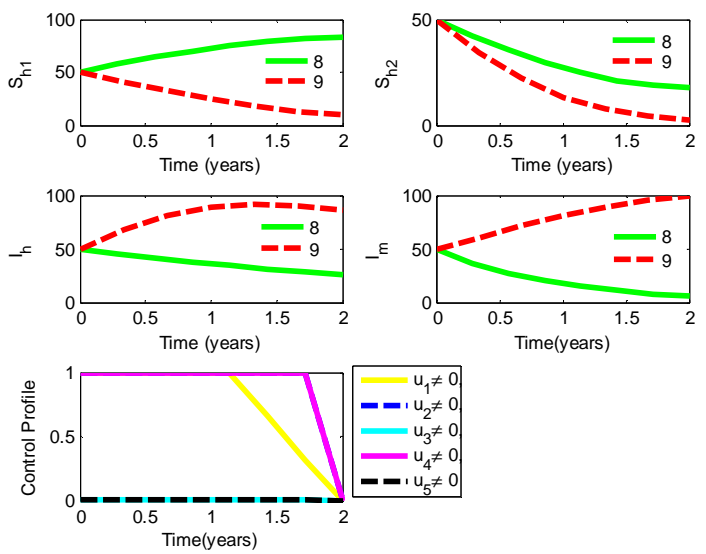

(viii)

Figure 2. (i)-(viii): Simulations of model system (3) showing the effects of optimal control strategies on the spread of dengue fever disease in the population.

infected human and mosquito population decreased.

The control profile for figure (ii), insecticides application $\left(u_{4}\right)$ is at upper bound for 1.714 years before dropping down to the lower bound while $u_{1}, u_{2}, u_{3}$ and $u_{5}$ are maintained at the lower bound till the final time.

Control profile for figure (iii), insecticides application $\left(u_{4}\right)$ and control maturation rate from larvae to adult $\left(u_{5}\right)$ are 
at upper bound for 1.714 years before dropping down to the lower bound, removing vector breeding areas $\left(u_{3}\right)$ is at 0.2828 and then drop down to the lower bound while $u_{1}$ and $u_{2}$ are maintained at the lower bound till the final time.

Control profile for figure (iv), insecticides application $\left(u_{4}\right)$ and control maturation rate from larvae to adult $\left(u_{5}\right)$ are at upper bound for 1.714 years and campaign for educating careless human susceptible $\left(u_{1}\right)$ is at upper bound for 0.5714 years before dropping down to the lower bound, removing vector breeding areas $\left(u_{3}\right)$ is at 0.2116 and then drop down to the lower bound while $u_{2}$ are maintained at the lower bound till the final time.

Control Profile for figure (v), shows that insecticides application $\left(u_{4}\right)$ is at upper bound for 1.714 years before dropping down to the lower bound while $u_{1}, u_{2}, u_{3}$ and $u_{5}$ are maintained at the lower bound till the final time.

Control profile for figure (vi) shows that control vectorhuman contact $\left(u_{2}\right)$ is at upper bound for 1.714 years before dropping down to the lower bound while $u_{1}, u_{3}$, $u_{4}$ and $u_{5}$ are at lower bound till the final time.

Control profile for figure (vii) shows that Campaign for educating careless human susceptible $\left(u_{1}\right)$ is at upper bound for 0.5714 years and control vector- human contact $\left(u_{2}\right)$ is at 1.714 years before dropping down to the lower bound while $u_{3}, u_{4}$ and $u_{5}$ are at lower bound till the final time.

Control profile for figure (viii) shows that Campaign for educating careless human susceptible $\left(u_{1}\right)$ is at upper bound for 1.143 years and insecticides application $\left(u_{4}\right)$ is at 1.714 years before dropping down to the lower bound while $u_{2}$, $u_{3}$ and $u_{5}$ are at lower bound till the final time.

\section{Cost Effectiveness Analysis}

We use Cost effectiveness analysis to determine the most cost effective strategy to use to control the disease. To achieve this purpose we need to compare the differences between the costs and health outcomes of these interventions. This is done by calculating the incremental cost-effectiveness ratio (ICER) which is generally described as the additional cost per additional health outcome. When comparing two or more competing intervention strategies incrementally, one intervention should be compared with the next-less-effective alternative [20].

The ICER numerator includes the differences in intervention costs, averted disease costs, costs of prevented cases and averted productivity losses if applicable. The ICER denominator is the differences in health outcomes [20]

We rank the strategies in increasing order of effectiveness, namely (1) when all controls are set to zero, (2) When insecticides application $\left(u_{4}\right)$ is used to optimize the objective function $(J)$ while other controls are set to zero, (3) When removing vector breeding areas $\left(u_{3}\right)$, insecticides application $\left(u_{4}\right)$ and control maturation rate from larvae to adult $\left(u_{5}\right)$ is used to optimize the objective function $(J)$ while other controls are set to zero, (4) When campaign to educate the careless human susceptible $\left(u_{1}\right)$, removing vector breeding areas $\left(u_{3}\right)$, insecticides application $\left(u_{4}\right)$ and control maturation rate from larvae to adult $\left(u_{5}\right)$ are used to optimize the objective function $(J)$ while control vectorhuman contact $\left(u_{2}\right)$ is set to zero, $(5)$ When control vectorhuman contact $\left(u_{2}\right)$ and insecticides application $\left(u_{4}\right)$ are used to optimize the objective function $(J)$ while other controls are set to zero, (6) When only control vector- human contact $\left(u_{2}\right)$ is used to optimize the objective function $(J)$ while other controls are set to zero, (7) When campaign to educate the careless human susceptible $\left(u_{1}\right)$ and control vector-human contact $\left(u_{2}\right)$ are used to optimize the objective function $(J)$ while other controls are set to zero and (8) When all controls are used to optimize the objective function $(J)$.

The difference between the total infectious individuals without control and the total infectious individuals with control is used to determine the "total number of infections averted" used are shown in table 1 of cost-effectiveness analysis [20]

Table 1. Ranking Control strategies in order of increasing effectiveness.

\begin{tabular}{|c|c|c|c|c|}
\hline Strategy & Control & Total infection averted & Total cost (\$) & $\mathbf{J}$ \\
\hline Strategyl & $u_{1}=u_{2}=u_{3}=u_{4}=u_{5}=0$ & 0 & 0 & 34682 \\
\hline Strategy 2 & $u_{4} \neq 0, u_{1}=u_{2}=u_{3}=u_{5}=0$ & 0.3273 & 8.9978 & 17749 \\
\hline Strategy3 & $u_{1}=0, u_{2}=0, u_{3} \neq 0, u_{4} \neq 0, u_{5} \neq 0$ & 0.363 & 25.3185 & 16416 \\
\hline Strategy 4 & $u_{1} \neq 0, u_{2}=0, u_{3} \neq 0, u_{4} \neq 0, u_{5} \neq 0$ & 0.4011 & 51.9736 & 16066 \\
\hline Strategy 5 & $u_{2} \neq 0, u_{4} \neq 0, u_{1}=u_{3}=u_{5}=0$ & 2.1229 & 63.9883 & 15670 \\
\hline Strategy6 & $u_{2} \neq 0, u_{1}=u_{3}=u_{4}=u_{5}=0$ & 2.1305 & 55.2799 & 22877 \\
\hline Strategy 7 & $u_{1} \neq 0, u_{2} \neq 0, u_{3}=u_{4}=u_{5}=0$ & 2.1305 & 90.8570 & 21754 \\
\hline Strategy8 & $u_{1} \neq u_{2} \neq u_{3} \neq u_{4} \neq u_{5} \neq 0$ & 2.1343 & 100.6025 & 14483 \\
\hline
\end{tabular}


Table 2. we exclude strategyl and compare strategy2and 3.

\begin{tabular}{lllll}
\hline Strategy & Control & Total infection averted & Total cost (\$) & J \\
\hline Strategy2 & $u_{4} \neq 0, u_{1}=u_{2}=u_{3}=u_{5}=0$ & 0.3273 & 8.9978 & 17749 \\
Strategy3 & $u_{1}=0, u_{2}=0, u_{3} \neq 0, u_{4} \neq 0, u_{5} \neq 0$ & 0.363 & 25.3185 & 16416 \\
\hline
\end{tabular}

This leads to the following values for the ICER,

$$
\begin{gathered}
\text { ICER } 2=\frac{8.9978}{0.3273}=27.49098686 \\
\text { ICER } 3=\frac{25.3185-8.9978}{0.363-0.3273}=457.162465
\end{gathered}
$$

The comparison between ICER (2) and ICER (3) shows a cost saving of $\$ 27.49098686$ for strategy2 over strategy 3 . The ICER for strategy 2 indicates that the strategy 3 is "strongly dominated". That is, strategy 3 is more costly and less effective than strategy2. Therefore, strategy3, the strongly dominated is excluded from the set of alternatives so that it does not consume limited resources.

Table 3. We exclude strategy 3 and compare strategies 2 and 4.

\begin{tabular}{lllll}
\hline Strategy & Control & Total infection averted & Total cost (\$) & J \\
\hline Strategy2 & $u_{4} \neq 0, u_{1}=u_{2}=u_{3}=u_{5}=0$ & 0.3273 & 8.9978 & 17749 \\
Strategy4 & $u_{1} \neq 0, u_{2}=0, u_{3} \neq 0, u_{4} \neq 0, u_{5} \neq 0$ & 0.4011 & 51.9736 & 16066 \\
\hline
\end{tabular}

This leads to the following values for the ICER,

$$
\begin{gathered}
\text { ICER } 2=\frac{8.9978}{0.3273}=27.49098686 \\
\text { ICER } 4=\frac{51.9736-8.9978}{0.4011-0.3273}=582.3279133
\end{gathered}
$$

cost saving of $\$ 27.49098686$ for strategy2 over strategy 4 . The ICER for strategy2 indicates the strategy4 is "strongly dominated". That is, strategy 4 is more costly and less effective than strategy2. Therefore, strategy 4, the strongly dominated is excluded from the set of alternatives so that it does not consume limited resources.

The comparison between ICER (2) and ICER (4) shows a

Table 4. We exclude strategy4 and compare strategies 2 and 5 .

\begin{tabular}{lllll}
\hline Strategy & Control & Total infection averted & Total cost (\$) & J \\
\hline Strategy2 & $u_{4} \neq 0, u_{1}=u_{2}=u_{3}=u_{5}=0$ & 0.3273 & 8.9978 & 17749 \\
Strategy5 & $u_{2} \neq 0, u_{4} \neq 0, u_{1}=u_{3}=u_{5}=0$ & 2.1229 & 63.9883 & 15670 \\
\hline
\end{tabular}

This leads to the following values for the ICER,

$$
\begin{gathered}
\text { ICER } 2=\frac{8.9978}{0.3273}=27.49098686 \\
\text { ICER } 5=\frac{63.9883-8.9978}{2.1229-0.3273}=30.62513923
\end{gathered}
$$

The comparison between ICER (2) and ICER (5) shows a cost saving of $\$ 27.49098686$ for strategy 2 over strategy5. The ICER for strategy 2 indicates the strategy 5 is "strongly dominated". That is, strategy 5 is more costly and less effective than strategy2.Therefore, strategy5, the strongly dominated is excluded from the set of alternatives so that it does not consume limited resources.

Table 5. We exclude strategy 5 and compare strategies 2 and 6.

\begin{tabular}{lllll}
\hline Strategy & Control & Total infection averted & Total cost (\$) & J \\
\hline Strategy2 & $u_{4} \neq 0, u_{1}=u_{2}=u_{3}=u_{5}=0$ & 0.3273 & 8.9978 & 17749 \\
Strategy6 & $u_{2} \neq 0, u_{1}=u_{3}=u_{4}=u_{5}=0$ & 2.1305 & 55.2799 & 22877 \\
\hline
\end{tabular}

This leads to the following values for the ICER,

$$
\begin{gathered}
\text { ICER } 2=\frac{8.9978}{0.3273}=27.49098686 \\
\text { ICER } 6=\frac{55.2799-8.9978}{2.1305-0.3273}=25.66664818
\end{gathered}
$$

The comparison between ICER (2) and ICER (6) shows a cost saving of $\$ 25.66664818$ for strategy 6 over strategy 2 . The ICER for strategy 6 indicates the strategy 2 is "strongly dominated". That is, strategy2 is more costly and less effective than strategy 6 . Therefore, strategy 2 , the strongly dominated is excluded from the set of alternatives so that it does not consume limited resources. 
Table 6. We exclude strategy2 and compare strategies6 and 7 .

\begin{tabular}{lllll}
\hline Strategy & Control & Total infection averted & Total cost (\$) & J \\
\hline Strategy6 & $u_{2} \neq 0, u_{1}=u_{3}=u_{4}=u_{5}=0$ & 2.1305 & 55.2799 & 22877 \\
Strategy7 & $u_{1} \neq 0, u_{2} \neq 0, u_{3}=u_{4}=u_{5}=0$ & 2.1305 & 90.8570 & 21754 \\
\hline
\end{tabular}

The comparison between strategy6 and strategy 7 shows a cost saving of $\$ 55.2799$ for strategy6 over strategy7. That is, strategy 7 is more costly than strategy6. Therefore, strategy7, the strongly dominated is excluded from the set of alternatives so that it does not consume limited resources.

Table 7. We exclude strategy 7 and compare strategies6 and 8.

\begin{tabular}{|c|c|c|c|c|}
\hline Strategy & Control & Total infection averted & Total cost $(\$)$ & $\mathbf{J}$ \\
\hline Strategy6 & $u_{2} \neq 0, u_{1}=u_{3}=u_{4}=u_{5}=0$ & 2.1305 & 55.2799 & 22877 \\
\hline Strategy8 & $u_{1} \neq u_{2} \neq u_{3} \neq u_{4} \neq u_{5} \neq 0$ & 2.1343 & 100.6025 & 14483 \\
\hline
\end{tabular}

This leads to the following values for the ICER,

$$
\begin{gathered}
\text { ICER } 6=\frac{55.2799-8.9978}{2.1305-0.3273}=25.66664818 \\
\text { ICER } 8=\frac{100.6025-55.2799}{2.1343-2.1305}=11940.15789
\end{gathered}
$$

The comparison between ICER (6) and ICER (8) shows a cost saving of $\$ 25.66664818$ for strategy6 over strategy 8 . The ICER for strategy6 indicates the strategy 8 is "strongly dominated". That is, strategy8 is more costly and less effective than strategy6.Therefore, strategy8, the strongly dominated is excluded from the set of alternatives so that it does not consume limited resources.

With this result, we therefore conclude that strategy6 (control vector-human contact $\left(u_{2}\right)$ ) is the most cost-effective of all the strategies for dengue fever disease control considered.

\section{Conclusion}

In this paper, the optimal control analysis for dengue fever model was performed using Pontryagin's maximum principle. Conditions for optimal control of the disease were derived and analysed with an effective use of campaign to educate the careless human susceptible $\left(u_{1}\right)$, control vector-human $\operatorname{contact}\left(u_{2}\right)$, removing vector breeding areas $\left(u_{3}\right)$, insecticides application $\left(u_{4}\right)$ and control maturation rate from larvae to adult $\left(u_{5}\right)$. The results suggest that the effective control vector- human contact $\left(u_{2}\right)$ strategy has a significant impact in reducing the dengue fever disease. From the cost-effectiveness analysis, the results suggest that control vector-human contact $\left(u_{2}\right)$ is the most cost-effective of all the strategies for dengue fever disease control considered.

\section{References}

[1] Rodrigues, H.S., Monteiro, M.T.T. and Torres, D.F.M. Dynamics of Dengue epidemics using optimal control. Mathematical and Computer Modelling, 2010, 1-10.
[2] Lenhart, S. and Workman, J.T. Optimal Control Applied to Biological Models, Mathematical and Computational Biology Series, Chapman and Hall/CRC, London, UK, 2007.

[3] WHO. Dengue and Dengue Haemorrhagic Fever. Fact Sheet No. 117. Geneva: World Health Organization, 2002

[4] Gibbons, R.V. and Vaughn, D.W. Dengue, an escalating problem. BMJ: British Medical Journal, 2002, 324: 1563.

[5] Seidu, B. and Makinde, O.D. Optimal Control of HIV/AIDS in the workplace in the Presence of Careless Individuals, Computational and Mathematical Methods in Medicine:2014:1-19.

[6] Ozair, M., Lashari, A.A., Jung, Il.H. and Okosun K.O. Stability Analysis and Optimal Control of a Vector-Borne Disease with Nonlinear Incidence. Discrete Dynamics in Nature and Society, 2012: 1-21.

[7] Thome, R.C.A., Yang, H.M. and Esteva, L. Optimal control of Aedes aegypti mosquitoes by the sterile insect technique and insecticide. Math. Biosci .2010, 223 :12-23.

[8] Rodrigues, H.S., Monteiro, M.T.T and Torres, D.F.M. Modeling and Optimal Control Applied to a Vector Borne Disease. International Conference on Computational and Mathematical Methods in Science and Engineering, CMMSE, 2012, 1063-1070.

[9] Rodrigues, H.S., Monteiro, M.T.T. and Torres, D.F.M.(2010). Insecticide control in a dengue epidemics model. In Numerical Analysis and Applied Mathematics, T. Simos, ed., AIP Conf. Proc.,2010, 1281(1):979-982.

[10] Rodrigues, H.S., Monteiro, M.T.T. and Torres, D.F.M. Dengue disease, basic reproduction number and control. International Journal of Computer Mathema tics ,2011, 1-13.

[11] Laarabi, H., Labriji, E.H., Rachik, M. and Kaddar, A. Optimal control of an epidemic model with a saturated incidence rate. Nonlinear Analysis: Modelling and Control, 2012, 17(4) 448-459

[12] El hia, M., Balatif, O., Rachik, M. and Bouyaghroumni, J. Application of optimal control theory to an SEIR model with immigration of infectives. International Journal of Computer Science Issues , 2013, 10 (2): 1694-0784

[13] Mwamtobe, P.M., Abelman ,S., Tchuenche,J.M. and Kasambara. Optimal (Control of) Intervention Strategies for Malaria Epidemic in Karonga District, Malawi. Abstract and Applied Analysis 2014: 1-20 
[14] Massawe, L.N., Massawe, E.S. and Makinde, O.D. Temporal model for dengue disease with treatment. Advances in Infectious Diseases, 2015, 5(1):1-16.

[15] Rodrigues HS, Monteiro MTT and Torres DFM. Sensitivity Analysis in a Dengue Epidemiological Model. Conference Papers in Mathematics Volume, 2013.

[16] Makinde, O.D. and Okosun, K.O. "Impact of chemo-therapy on optimal control of malaria disease with infected immigrants," BioSystems, 2011, 104 (1): 32-41.

[17] Pontryagin, L.S., Boltyanskii, V.G., Gamkrelidze, R.V. and Mishchenko, E.F. The Mathematical Theory of Optimal Processes. Wiley, New York,1962.
[18] W.H. Fleming and R.W. Rishel, Deterministic and Stochastic Optimal Control, vol.1, Springer, New York, NY, USA, 1975.

[19] Dumont Y, Chiroleu F and Domerg C. "On a temporal model for the Chikungunya disease: modelling, theory and numerics," Mathematical Biosciences, 2008, 213(1): 80-91.

[20] Okosun K.O. O.D. Makinde, I. Takaidza. Impact of optimal control on the treatment of HIV/AIDS and screening of unaware infectives, Applied Mathematical Modelling , 2013, 37: $3802-3820$. 